\title{
Supporting information for: \\ Synthesis, Properties, and Derivatization of \\ Poly(dihydrogermane): A Germanium-Based Polyethylene \\ Analogue
}

Haoyang Yu, § Chuyi Ni, § Alyxandra N. Thiessen, Ziqi Li and Jonathan G.C. Veinot* ${ }^{*} \dagger$

$\S \mathrm{H}$. Yu and C. Ni contributed equally to this work.

Department of Chemistry, University of Alberta, 11227 Saskatchewan Drive, Edmonton, Alberta Canada T6G 2G2

\author{
E-mail: jveinot@ualberta.ca
}




\section{Table of Figures}

Figure S1. Measured (black) and fitted (red) powder XRD patterns of $\mathrm{CaGe}$ using $\lambda=$ $0.68745 \AA$. Primary phase is $97 \% \mathrm{CaGe}$ and secondary phase is \#\% $\mathrm{CaGe}_{2} \ldots \ldots . . . . .3$

Figure S2. Photograph of (a) freshly ground crystalline $\mathrm{CaGe}$, and (b) $\mathrm{CaGe}$ powder after ca. 1 min exposure to ambient conditions.

Figure S3. A photograph obtained using an optical microscopy equipped at 20x magnification. $\left(\mathrm{GeH}_{2}\right)_{n}$ prepared by Method 1 (a) before and (b) after $50 \%$ laser power $(1.99 \mathrm{~mW})$ exposure for $60 \mathrm{~s}$.

Figure S4. FTIR spectra of Method $1\left(\mathrm{GeH}_{2}\right)_{n}$ products at different stages: (a) $\left(\mathrm{Ca}(\mathrm{OH})_{2} \mathrm{GeH}_{2}\right)_{n}$ and (b) $\left(\mathrm{GeH}_{2}\right)_{n}$ with adsorbed $\mathrm{H}_{2} \mathrm{O}_{-}$ 4

Figure S5. Dynamic light scattering hydrodynamic diameter output of (a) $\left(\mathrm{GeH}_{2}\right)_{n}$ and (b) of $\left(\mathrm{Ge}\left(\mathrm{C}_{12} \mathrm{H}_{25}\right)_{2}\right)_{n}$ prepared by both methods.

Figure S6. Survey XP spectra of (a) Ar plasma cleaned Ge (111) wafer, (b) CaGe after Ar plasma cleaning, $\left(\mathrm{GeH}_{2}\right)_{n}$ prepared by method 2, (c) before and (d) after $\mathrm{Ar}$ plasma cleaning.

Figure S7. High-resolution XP spectra of CaGe after etching, (top) C 1s region and (bottom) $\mathrm{Ca} 2 \mathrm{p}$ region. 7

Figure S8. Schematic illustration of [020], [200] and [021] reflection................................

Figure S9. EDX spectrum of $\left(\mathrm{GeH}_{2}\right)_{n}$ (method 2), measured at area with vacuum background on a holey carbon grid...................................................................................8

Figure S10. EDX spectrum of dodecyl-functionalized polygermane (method 2), measured at area with vacuum background on a holey carbon grid.

Figure S11. HAADF-STEM images of $\left(\mathrm{Ge}\left(\mathrm{C}_{12} \mathrm{H}_{25}\right)_{2}\right)_{n}$ prepared using $\left(\mathrm{GeH}_{2}\right)_{n}$ obtained from Method 1. (a, b) without sonication: (a) low-resolution image, (b) high-resolution image; (c, d) the assembly of freestanding $\left(\mathrm{Ge}\left(\mathrm{C}_{12} \mathrm{H}_{25}\right)_{2}\right)_{n}$ at different magnification.

Figure S12. HAADF-STEM images of $\left(\mathrm{Ge}\left(\mathrm{C}_{12} \mathrm{H}_{25}\right)_{2}\right)_{n}$ prepared using $\left(\mathrm{GeH}_{2}\right)_{n}$ obtained from Method 2 after plasma cleaning. (a) residual large strands and (b) freestanding assembly after harsh cleaning; ultra-high magnification images of freestanding assembly after (c) gentle cleaning and (d) harsh cleaning. ...........................................11

Figure S13. (a) DRA measurement, (b) Raman spectra, (c) high-resolution Ge 3d XP spectra and (d) high-resolution $\mathrm{C}$ 1s XP spectra of $\left(\mathrm{Ge}\left(\mathrm{C}_{12} \mathrm{H}_{25}\right)_{2}\right)_{n}$ prepared from using $\left(\mathrm{GeH}_{2}\right)_{\mathrm{n}}$ obtained using Methods 1 and 2 . 


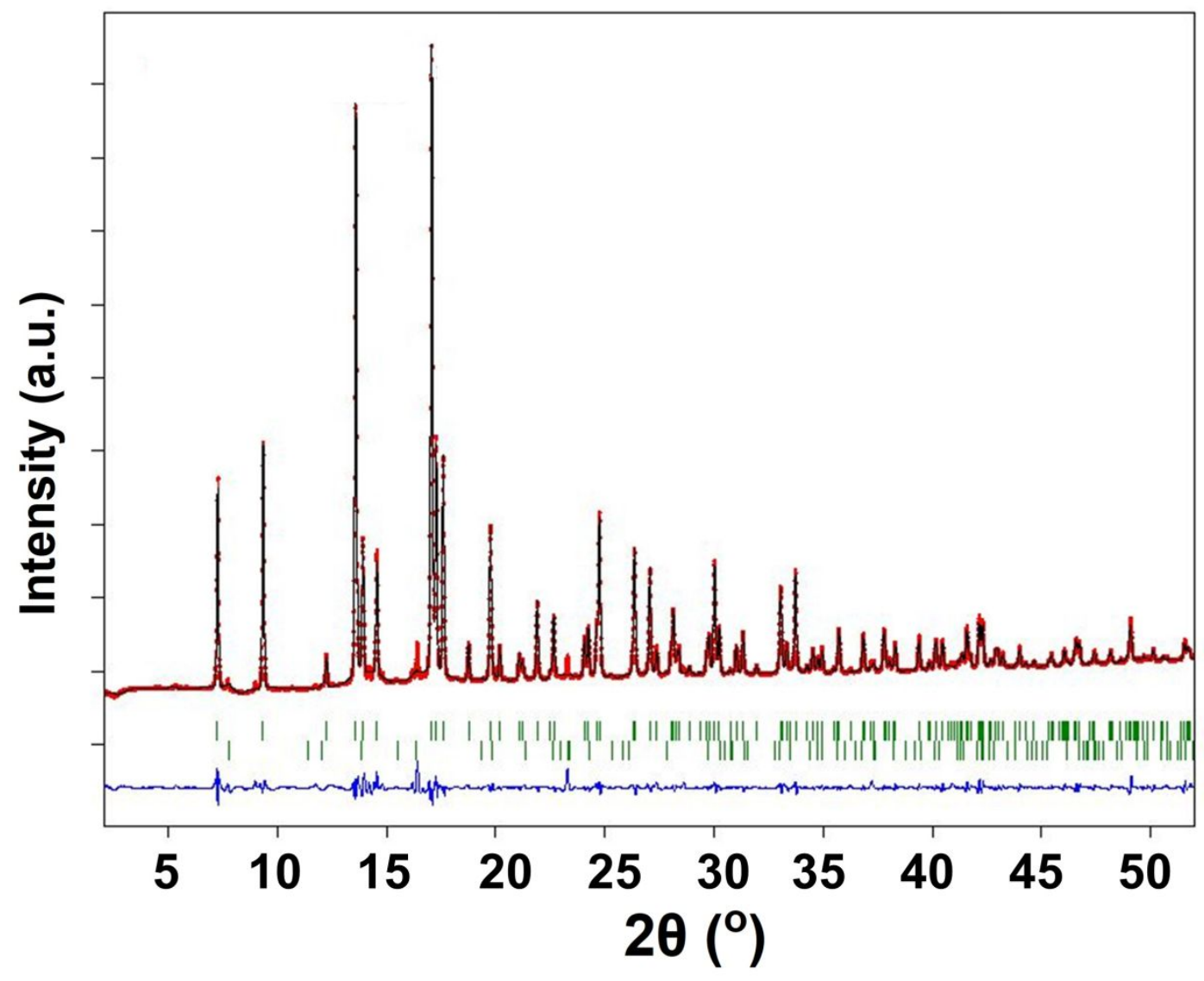

Figure S1. Measured (black) and fitted (red) powder XRD patterns of CaGe using $\lambda=$ $0.68745 \AA$. Primary phase is $97 \% \mathrm{CaGe}$ and secondary phase is $3 \% \mathrm{CaGe}_{2}$.

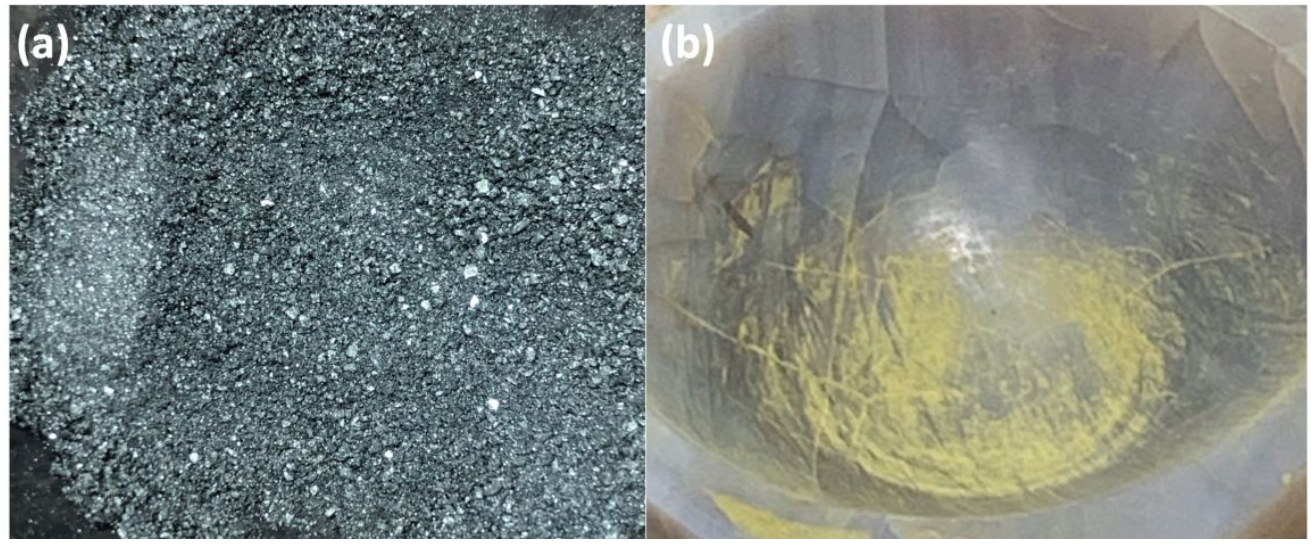

Figure S2. Photograph of (a) freshly ground crystalline CaGe, and (b) CaGe powder after ca. 1 min exposure to ambient conditions. 

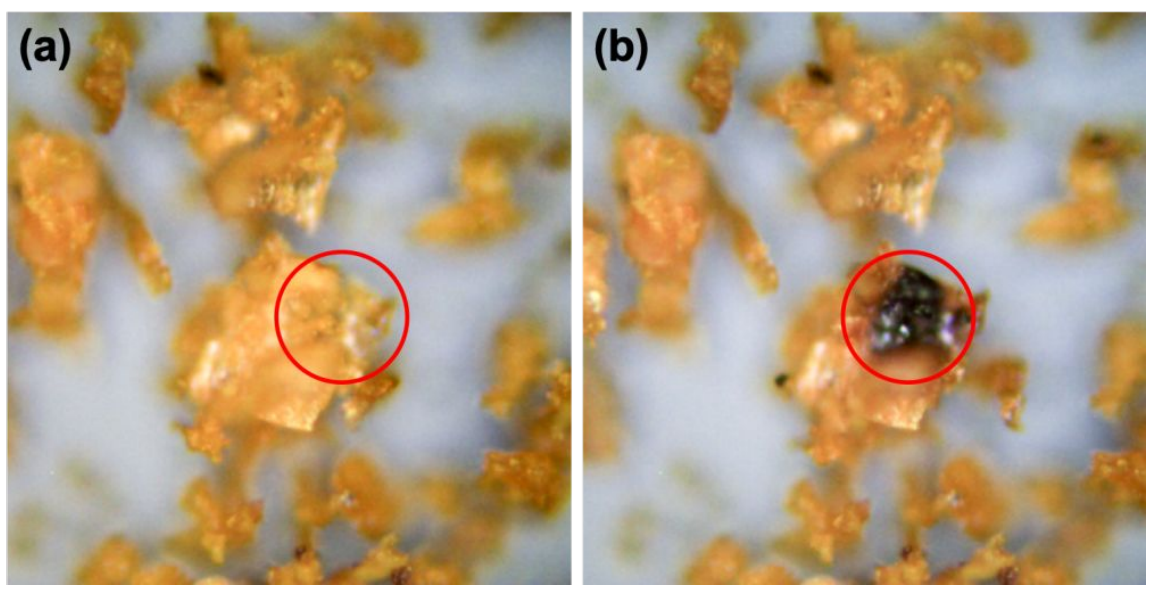

Figure S3. A photograph obtained using an optical microscopy equipped at 20x magnification. $\left(\mathrm{GeH}_{2}\right)_{n}$ prepared by Method 1 (a) before and (b) after $50 \%$ laser power $(1.99 \mathrm{~mW})$ exposure for $60 \mathrm{~s}$.

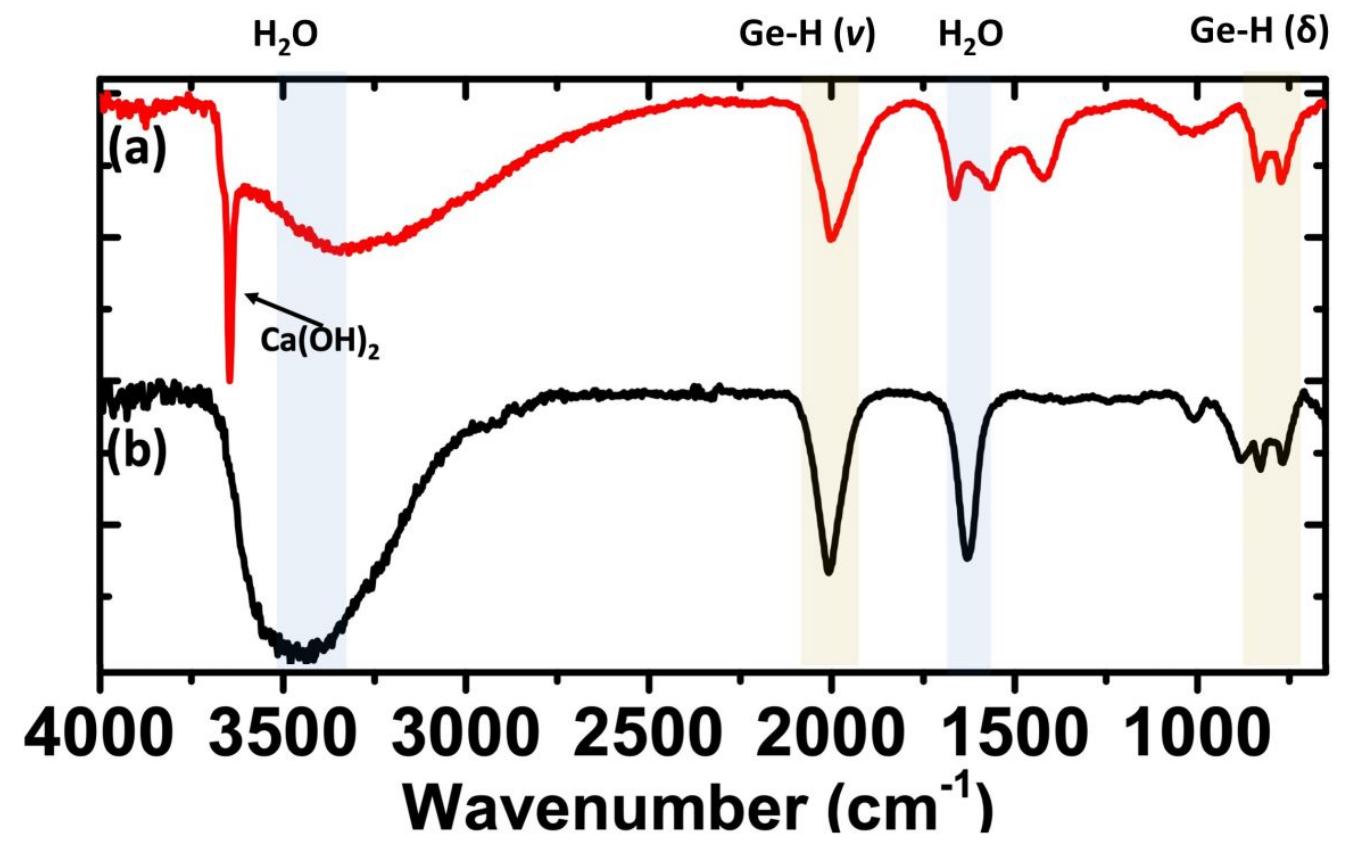

Figure S4. FTIR spectra of Method $1\left(\mathrm{GeH}_{2}\right)_{n}$ products at different stages: (a) $\left(\mathrm{Ca}(\mathrm{OH})_{2} \mathrm{GeH}_{2}\right)_{n}$ and $(\mathrm{b})\left(\mathrm{GeH}_{2}\right)_{n}$ with adsorbed $\mathrm{H}_{2} \mathrm{O}$ - 
(a)
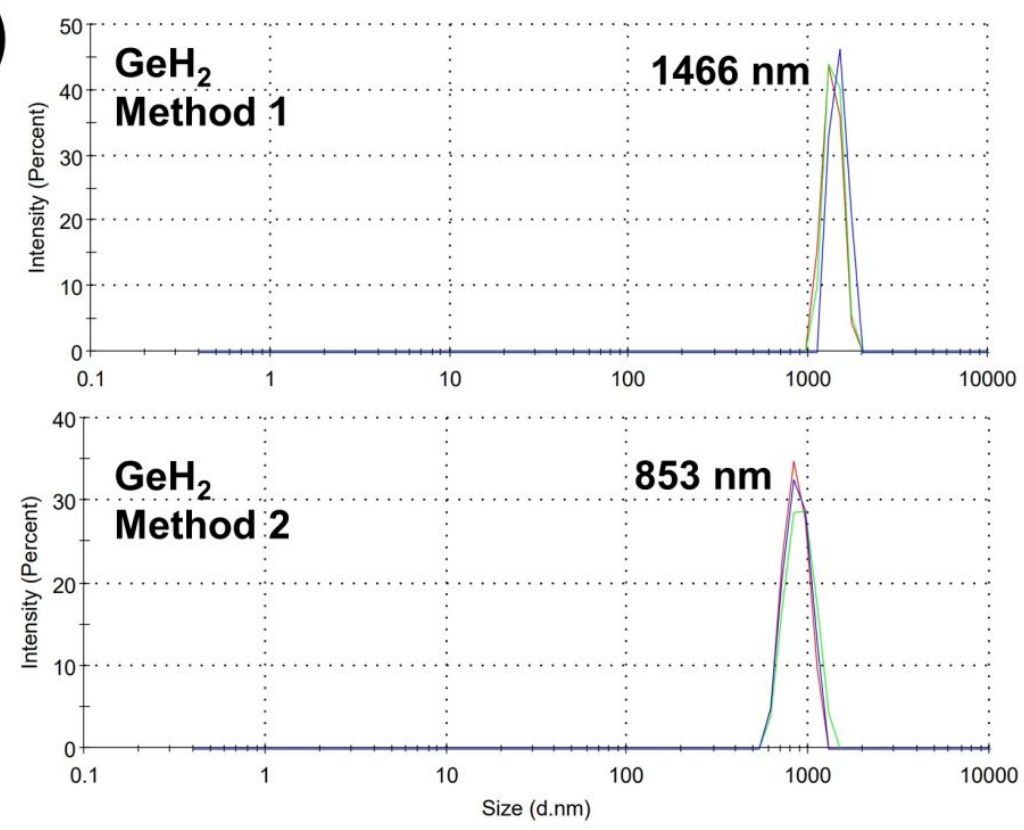

(b)
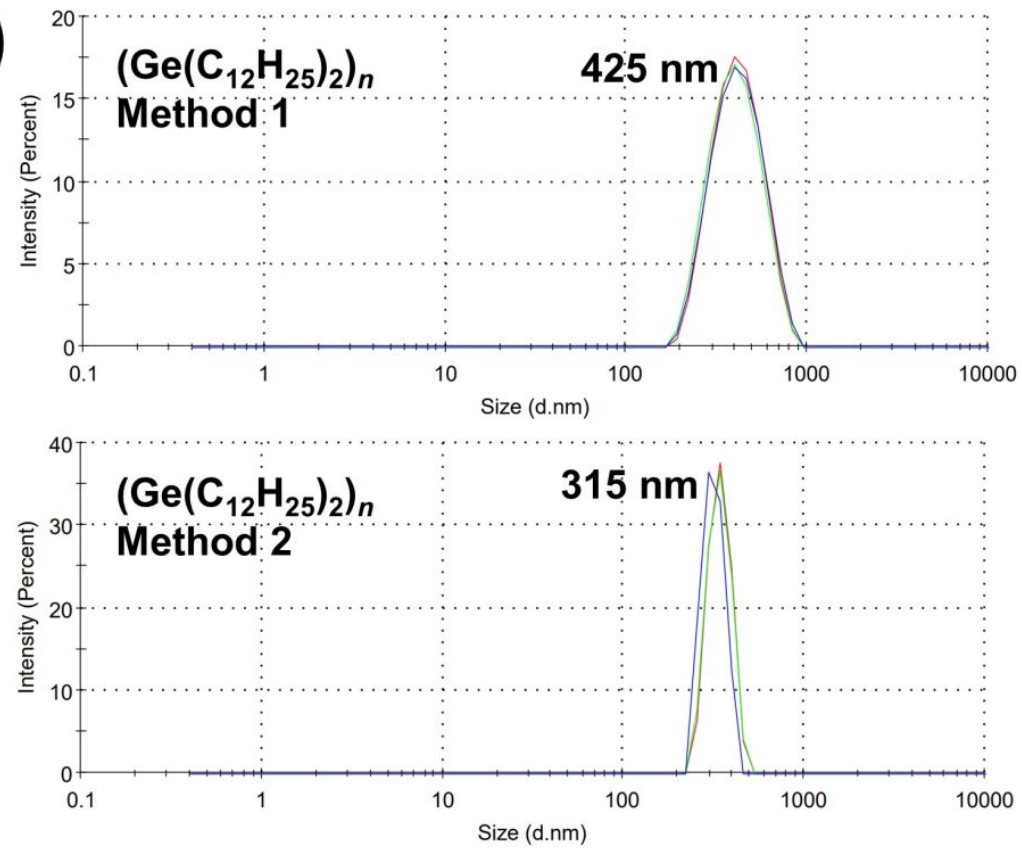

Figure S5. Dynamic light scattering hydrodynamic diameter output of $(\mathrm{a})\left(\mathrm{GeH}_{2}\right)_{n}$ and (b) of $\left(\mathrm{Ge}\left(\mathrm{C}_{12} \mathrm{H}_{25}\right)_{2}\right)_{n}$ prepared by both methods. 


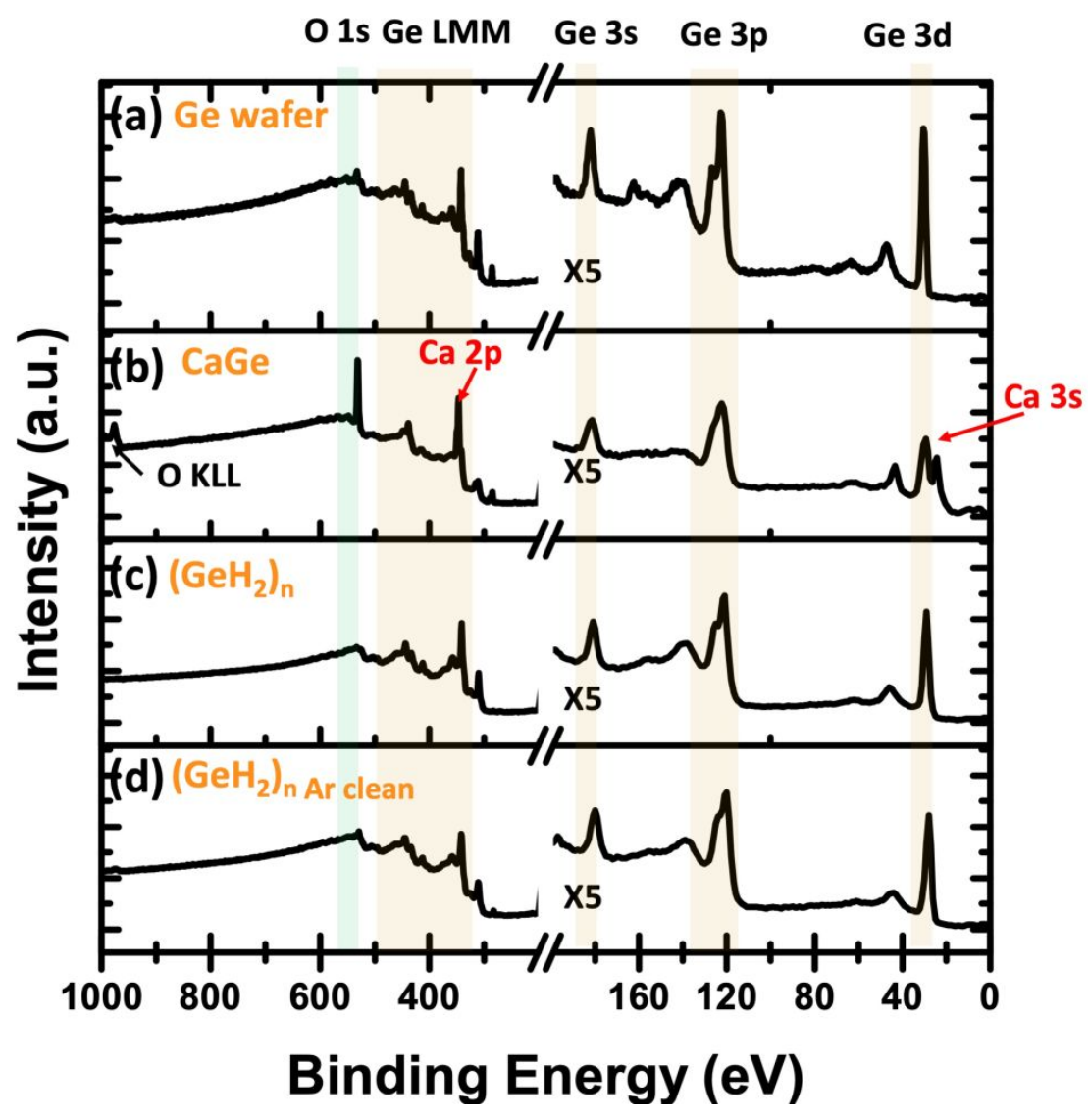

Figure S6. Survey XP spectra of (a) Ar plasma cleaned Ge (111) wafer, (b) CaGe after Ar plasma cleaning, $\left(\mathrm{GeH}_{2}\right)_{n}$ prepared by method 2, (c) before and (d) after Ar plasma cleaning. 

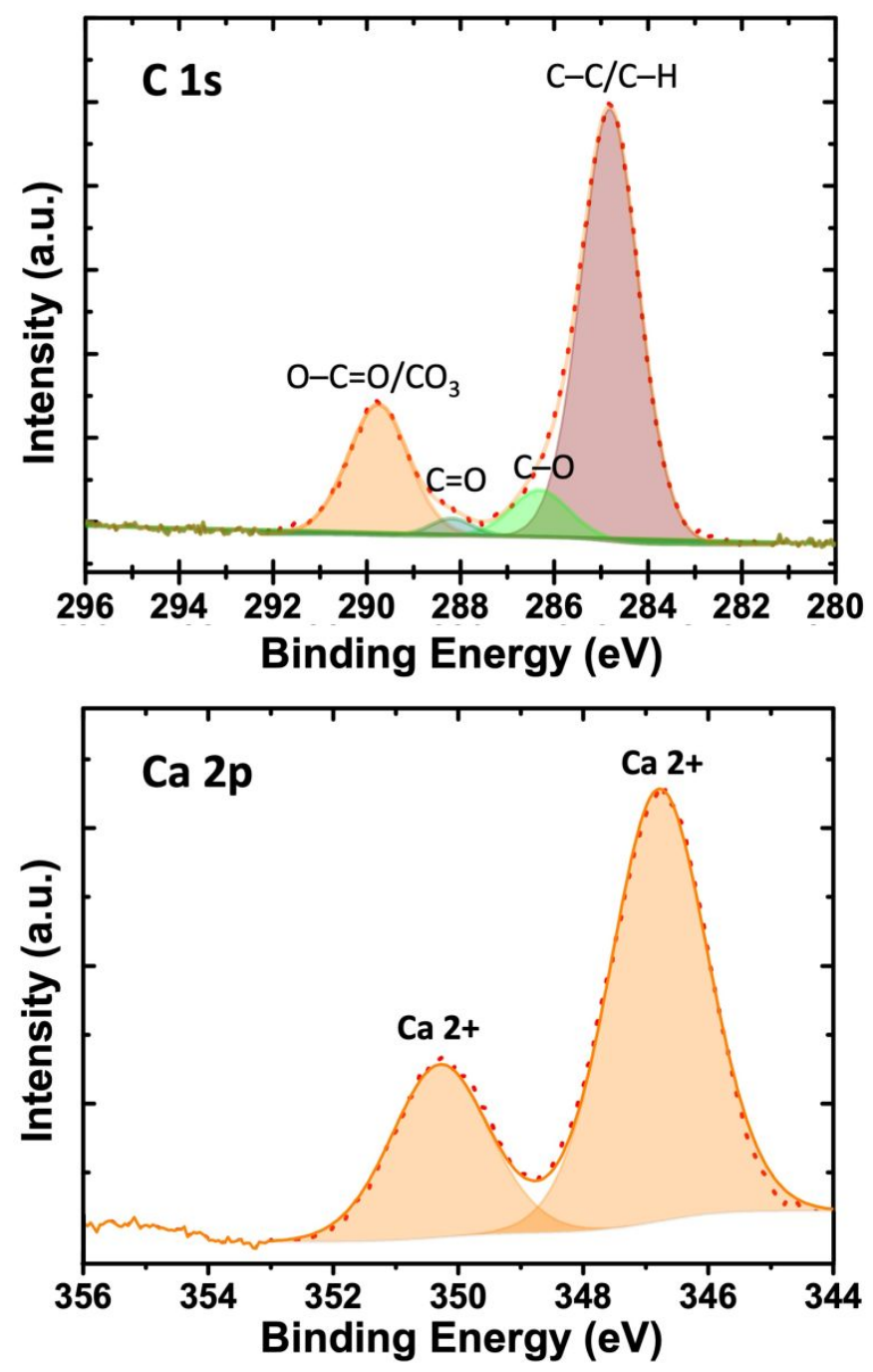

Figure S7. High-resolution XP spectra of $\mathrm{CaGe}$ after etching, (top) $\mathrm{C}$ 1s region and (bottom) Ca $2 p$ region. 

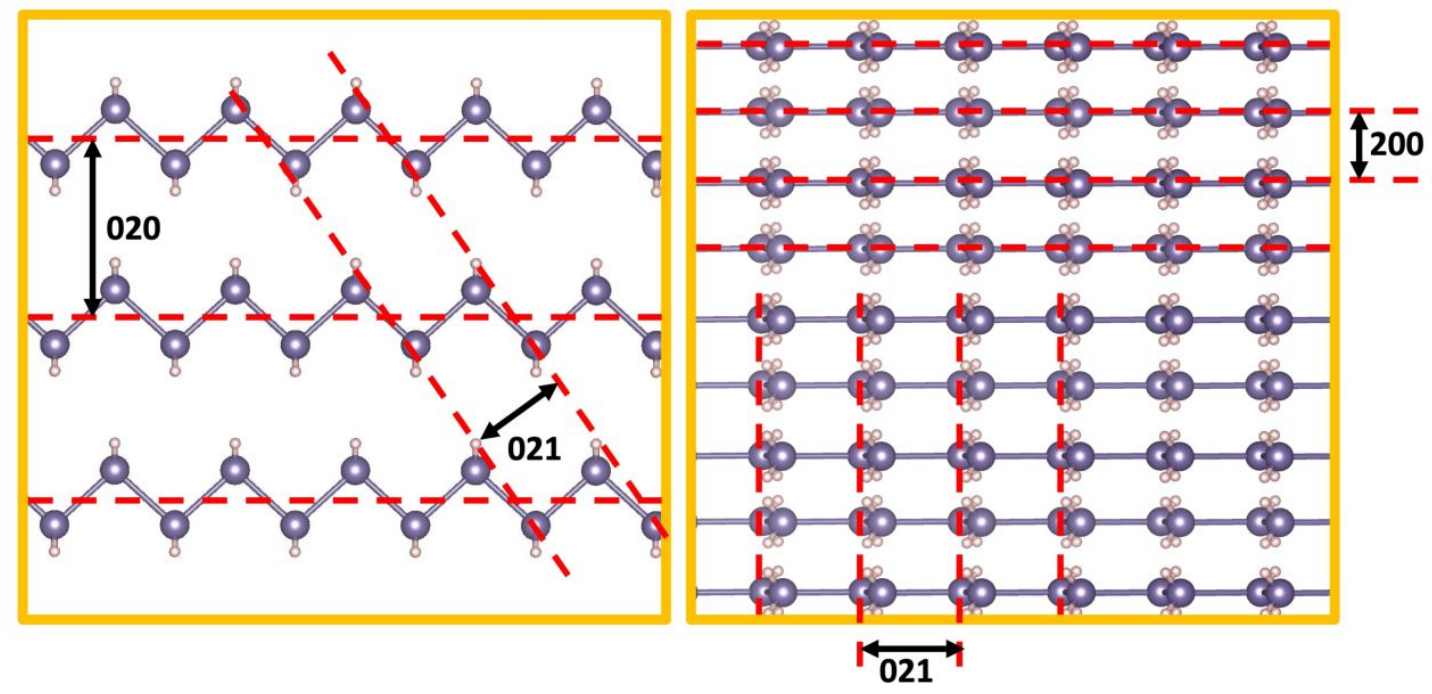

Figure S8. Schematic illustration of CaGe [020], [200] and [021] reflection.

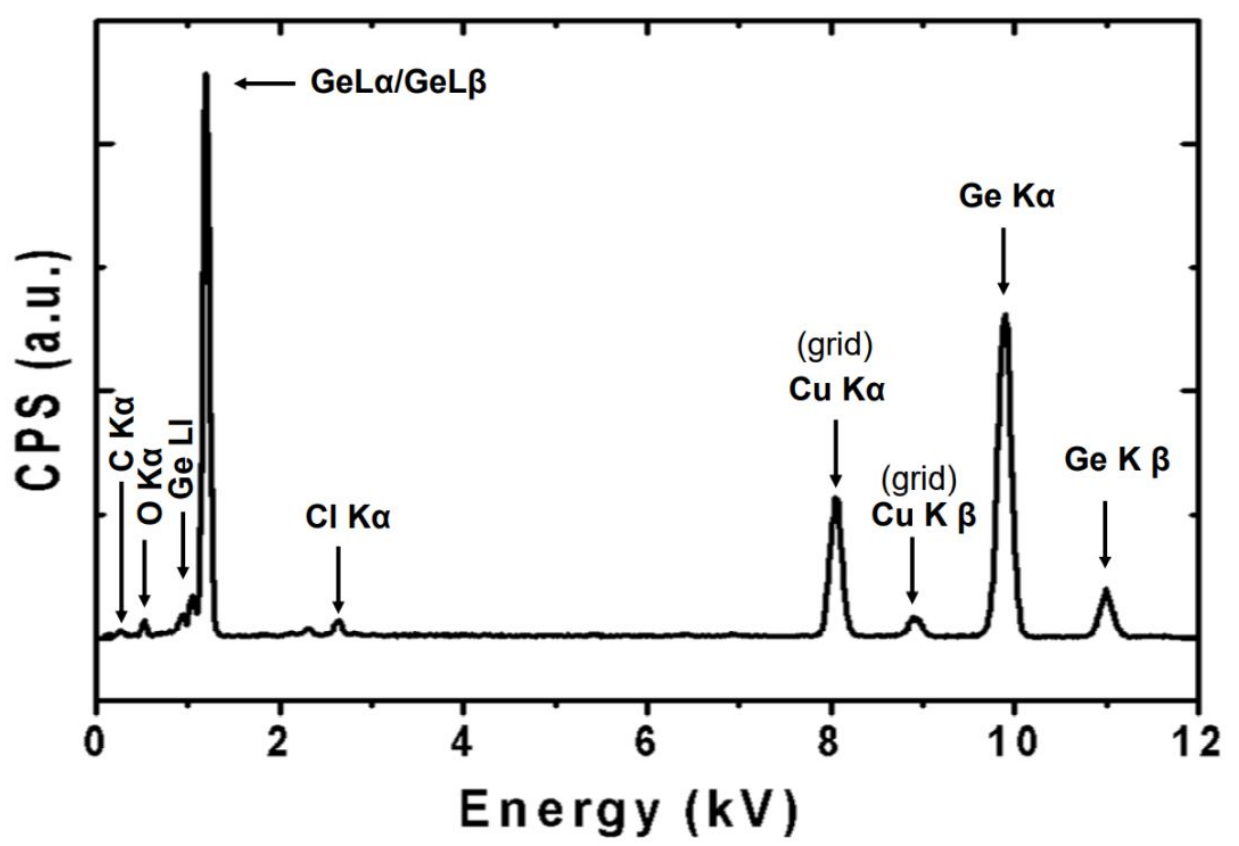

Figure S9. EDX spectrum of $\left(\mathrm{GeH}_{2}\right)_{n}$ prepared using $\left(\mathrm{GeH}_{2}\right)_{\mathrm{n}}$ obtained from Method 2, measured at area with vacuum background on a holey carbon grid. 


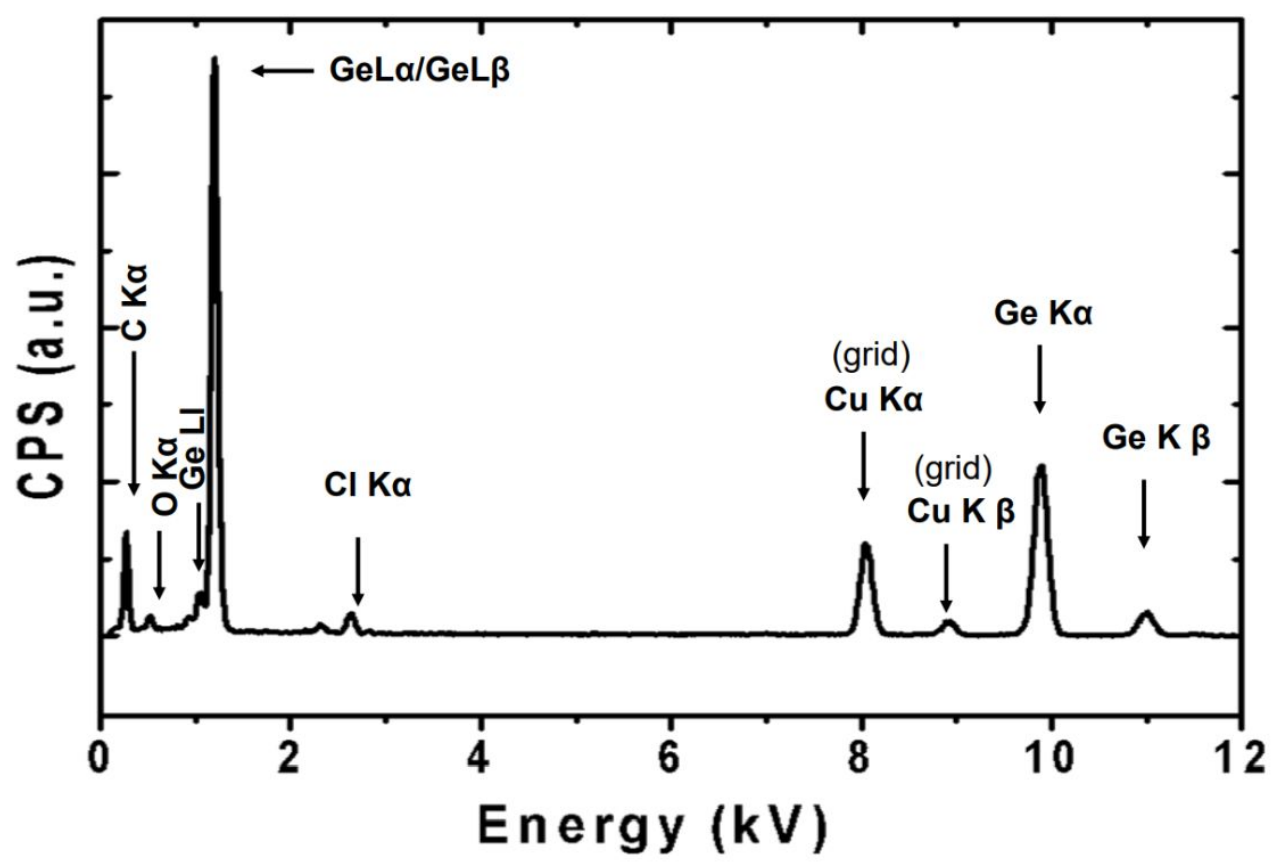

Figure S10. EDX spectrum of dodecyl-functionalized polygermane prepared using $\left(\mathrm{GeH}_{2}\right)_{\mathrm{n}}$ obtained from Method 2, measured at area with vacuum background on a holey carbon grid. 


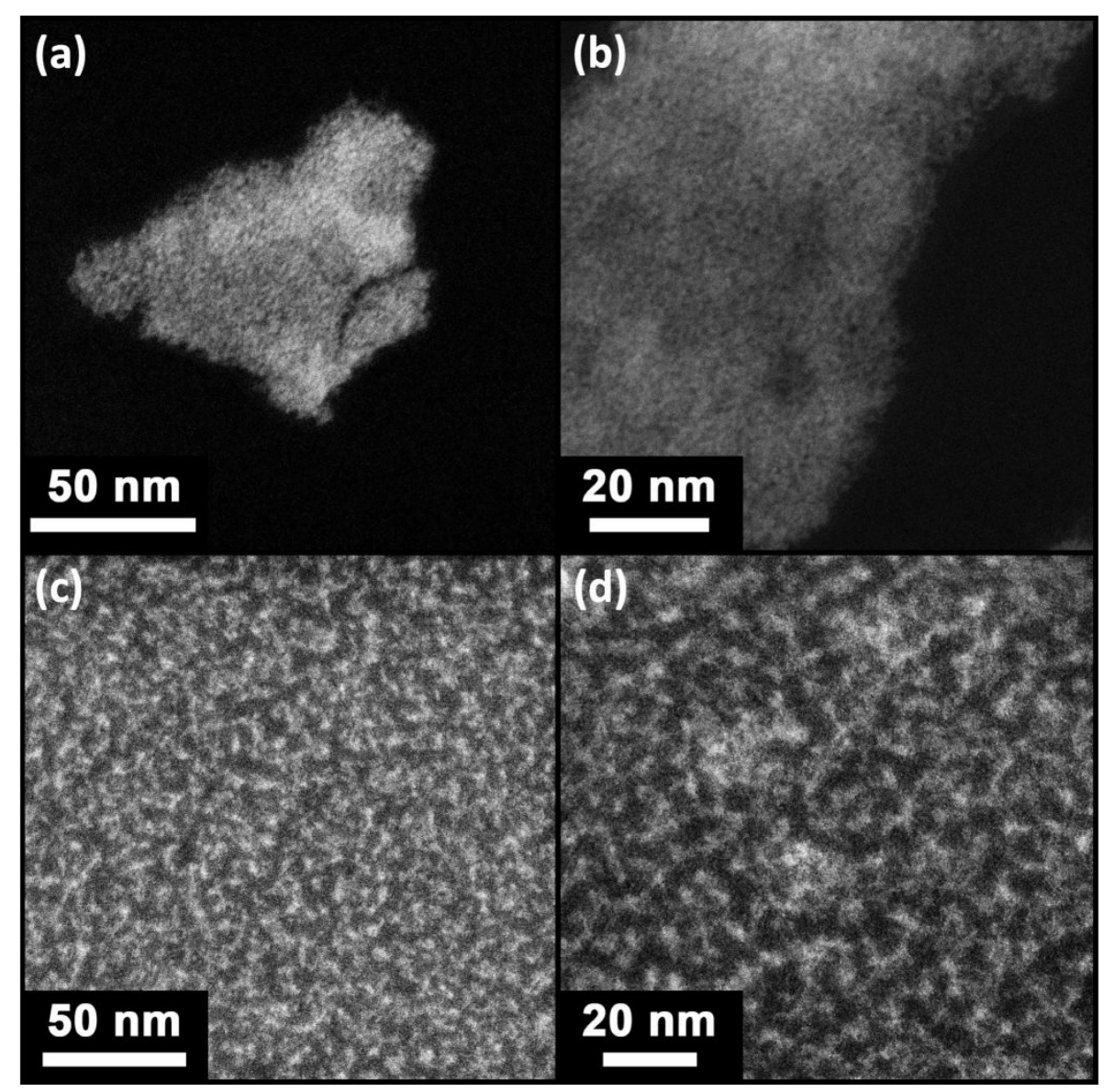

Figure S11. HAADF-STEM images of $\left(\mathrm{Ge}\left(\mathrm{C}_{12} \mathrm{H}_{25}\right)_{2}\right)_{n}$ prepared using $\left(\mathrm{GeH}_{2}\right)_{n}$ obtained from Method 1. (a, b) without sonication: (a) low-resolution image, (b) high-resolution image; (c, d) the assembly of freestanding $\left(\mathrm{Ge}\left(\mathrm{C}_{12} \mathrm{H}_{25}\right)_{2}\right)_{n}$ at different magnification. 


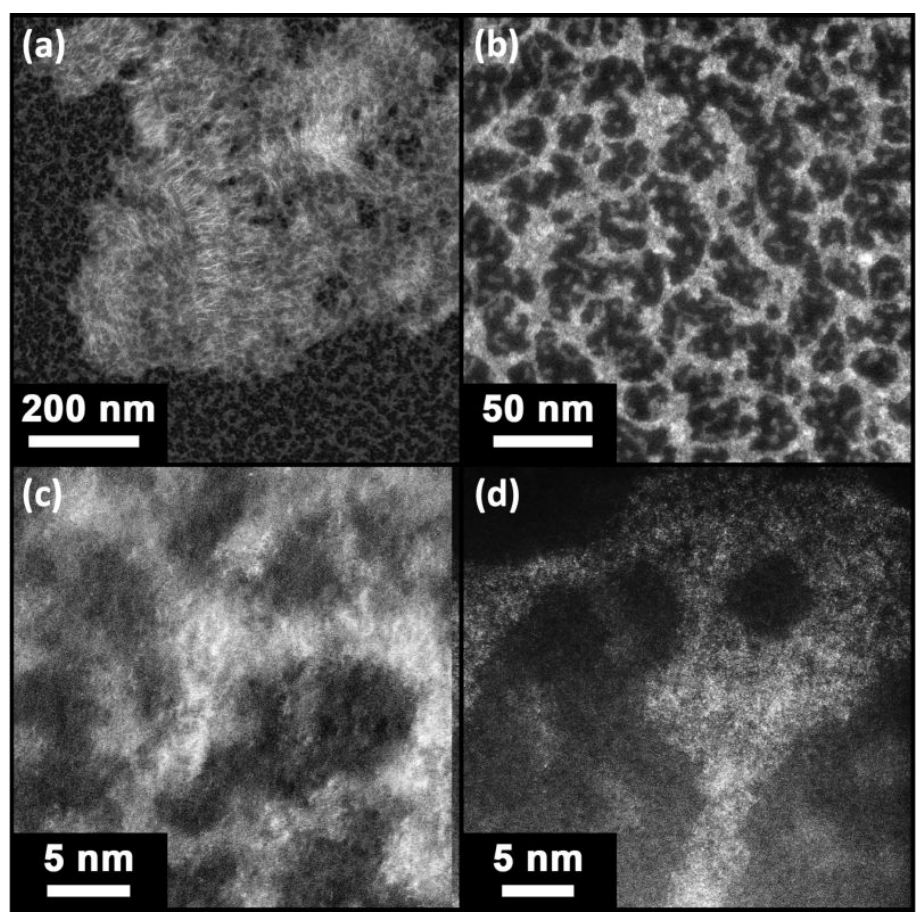

Figure S12. HAADF-STEM images of $\left(\mathrm{Ge}\left(\mathrm{C}_{12} \mathrm{H}_{25}\right)_{2}\right)_{n}$ prepared using $\left(\mathrm{GeH}_{2}\right)_{n}$ obtained from Method 2 after plasma cleaning. (a) residual large strands and (b) freestanding assembly after harsh cleaning; ultra-high magnification images of freestanding assembly after (c) gentle cleaning and (d) harsh cleaning. 

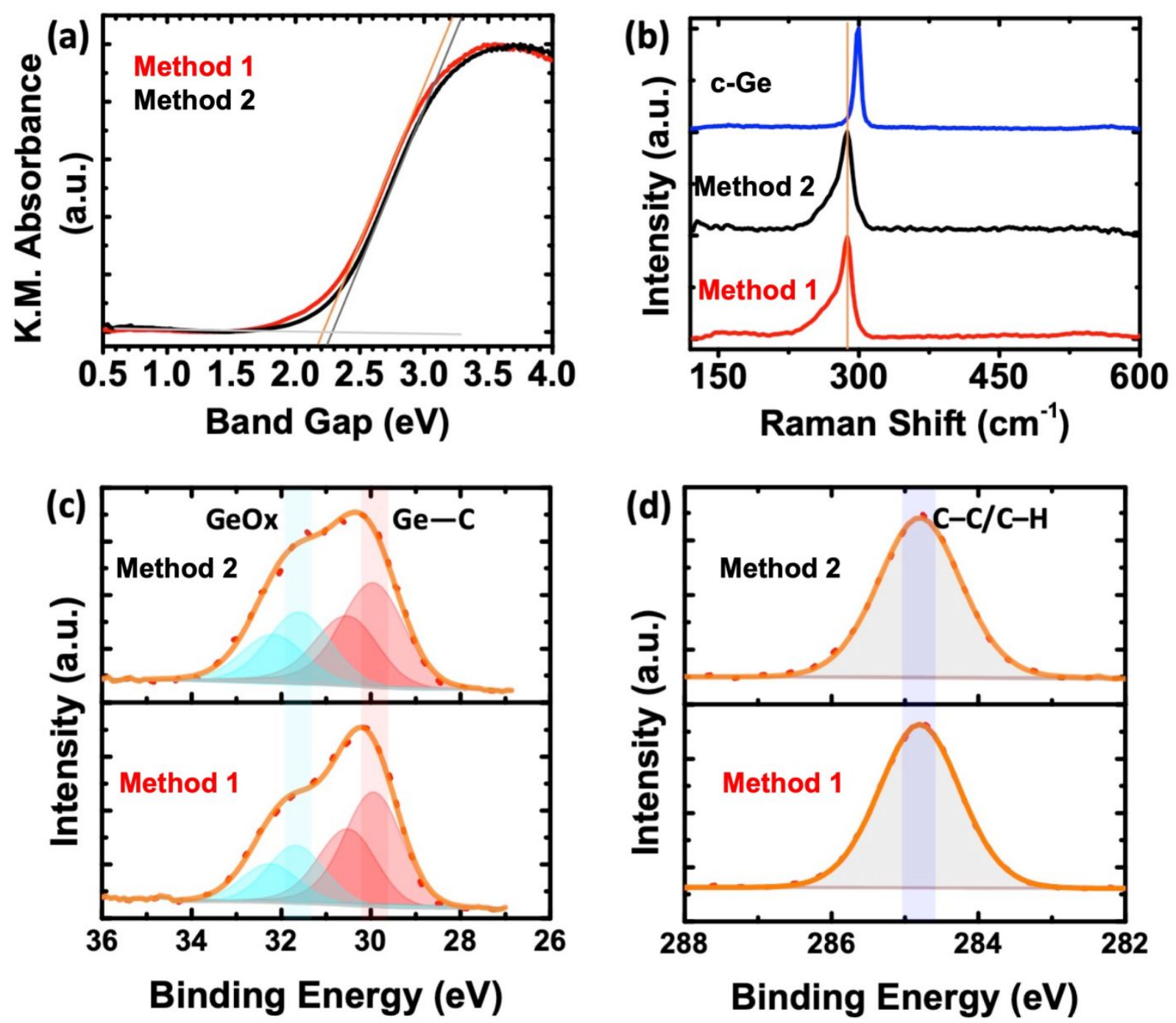

Figure S13. (a) DRA measurement, (b) Raman spectra, (c) high-resolution Ge 3d XP spectra and (d) high-resolution $\mathrm{C}$ 1s XP spectra of $\left(\mathrm{Ge}\left(\mathrm{C}_{12} \mathrm{H}_{25}\right)_{2}\right)_{n}$ prepared using $\left(\mathrm{GeH}_{2}\right)_{n}$ obtained from Methods 1 and 2. 